\title{
Reconsidering Calcium Dehydration as the Rate-Determining Step in Calcium Mineral Growth
}

\author{
Janou A. Koskamp, ${ }^{\dagger}$ Sergio E. Ruiz-Hernandez, ${ }^{\dagger}$ Devis Di Tommaso, ${ }^{\ddagger}$ Alin Marin Elena, ${ }^{\S}$ \\ Nora H. De Leeuw, ${ }^{\dagger, \|}$ and Mariette Wolthers, ${ }^{\dagger}{ }^{\dagger}$ \\ ${ }^{\dagger}$ Department of Earth Sciences-Geochemistry, Utrecht University, 3584 CB Utrecht, The Netherlands \\ ${ }^{*}$ Materials Research Institute and School of Biological and Chemical Sciences, Queen Mary University of London, Mile End Road, \\ E1 4NS London, United Kingdom \\ ${ }^{\S}$ Daresbury Laboratory, STFC, Daresbury WA4 4AD, United Kingdom \\ "School of Chemistry, Cardiff University, Main Building Park Place, Cardiff CF10 3AT, United Kingdom
}

Supporting Information

\begin{abstract}
The dehydration of cations is generally accepted as the rate-limiting step in many processes. Molecular dynamics (MD) can be used to investigate the dynamics of water molecules around cations, and two different methods exist to obtain trajectory-based water dehydration frequencies. Here, these two different post-processing methods (direct method versus survival function) have been implemented to obtain calcium dehydration frequencies from a series of trajectories obtained using a range of accepted force fields. None of the method combinations reproduced the commonly accepted experimental water exchange frequency of $10^{-8.2} \mathrm{~s}^{-1}$. Instead, our results suggest much faster water dynamics, comparable with more accurate ab initio MD simulations and with experimental values obtained using neutron scattering techniques. We obtained the best agreement using the survival function method to characterize the water dynamics, and we show that different method combinations significantly affect the outcome. Our work strongly suggests that the fast water exchange kinetics around the calcium ions is not rate-limiting for reactions involving dissolved/solvated calcium. Our results further suggest that, for alkali and most of the earth alkali metals, mechanistic rate laws for growth, dissolution, and adsorption, which are based on the principle of rate-limiting cation dehydration, need careful reconsideration.
\end{abstract}

\section{INTRODUCTION}

The dynamics of water molecules surrounding reacting cations is generally considered as rate-determining for the reactions involving these ions in aqueous solutions. ${ }^{1-6}$ Almost 60 years ago, ultrasonic sound adsorption experiments performed by Eigen indicated a large range of water exchange frequencies around cations. ${ }^{7,8}$ Years later, Nielsen found a correlation between the frequency of ions entering surface kink sites and the dehydration frequencies of the cations in this surface where the attachment frequency of the ions was approximately $10^{-3}$ times its dehydration frequency. Nielsen proposed that this difference was due to the ion diffusion from solution to a kink site, a process included in his overall attachment frequency. Nevertheless, as anions dehydrate faster, cations are considered to control the reaction rate. ${ }^{9-11}$ These general findings have been incorporated into mechanistic models explaining crystal dissolution (e.g., Lasaga and Lüttge $)^{12,13}$ and crystal growth. $^{14-22}$ Essentially, in such models, it is assumed that the exchange frequency of water molecules between the first hydration shell around cation surface sites and bulk water controls the reaction rate.

The dynamics of water molecules can be investigated using molecular dynamics (MD) simulations, for example, of water molecules around dissolved ions, ${ }^{3,23-25}$ at mineral-water interfaces, ${ }^{26-29}$ and in amorphous materials. ${ }^{30}$ Several studies have employed MD to study ion complexation and dynamics in water, ${ }^{31-34}$ whereas the technique has also been used to analyze adsorption of small organics on mineral-water interfaces, ${ }^{30,35}$ the structural changes in (water near) mineral interfaces, $^{36,37}$ and the influence of ion impurities on the kinetic stability of amorphous materials. ${ }^{38}$

In the case of calcite, combined experimental and computational work has shown that electrolyte ions impact water exchange frequencies and the crystal growth rate. ${ }^{3} \mathrm{MD}$ simulations also confirmed that the formation of the kink sites on the dissolving edge of the obtuse step of calcite is the rate-limiting step and the dissolution of this edge is thermodynamically favored $^{39}$ and the addition of the first units of calcium carbonate is enthalpically unfavorable. ${ }^{40}$ Also, there is good agreement in the description of the structure of water at the calcite-water interface, as observed computationally, ${ }^{41,42}$ using force-modulated atomic force microscopy, ${ }^{43,44}$ small-angle X-ray reflectivity, ${ }^{42}$ and neutron diffraction. ${ }^{45}$

Despite the promising agreement on the structure of the calcite-water interface obtained computationally and experimentally, large discrepancies remain in observations of water

Received: July 5, 2019

Revised: October 15, 2019

Published: October 16, 2019 
dynamics. Recent studies using ab initio MD (AIMD) calculations ${ }^{46,47}$ show that exchange frequencies around dissolved calcium are almost 3 orders of magnitude higher than Eigen's value. Comparably high frequencies have also been observed around dissolved calcium using incoherent quasi-elastic neutron scattering (IQUENS), ${ }^{48}$ from which the authors concluded that the water exchange frequency around calcium ions is faster than $10^{10} \mathrm{~s}^{-1}$. Other MD simulations have indicated frequencies much closer to those measured by Eigen, ${ }^{28}$ while classical MD simulations have resulted in water exchange frequencies around dissolved calcium and calcium in calcite surfaces ${ }^{37}$ that are similar to those observed in ab initio MD and IQUENS. Besides, it is still debated if dehydration of the attaching species is rate-limiting in crystal growth ${ }^{5}$ or that step-migration of carbonate ions is rate-limiting for kink nucleation. $^{49}$

While an evaluation of the differences between the various experimentally observed frequencies is beyond the scope of the paper, the large range in computationally determined water exchange frequencies may be explained by differences between the force fields used in $\mathrm{MD}$ methods and/or the approach followed to obtain exchange frequencies from the MD output. Here, we present our evaluation of both potential areas for discrepancy. We have simulated identical systems of dissolved calcium using four sets of force fields, some of which have previously provided contradictory results. We next evaluated two generally accepted methods to determine water exchange frequencies. The results from our simulations and calculations provide new insights into the impact of the water dynamics around calcium on the reactivity of materials containing this cation. Our results dispute that calcium dehydration is the ratelimiting step in mineral formation and dissolution and suggest that other processes are rate-determining instead. We discuss implications for mechanistic growth models.

\section{COMPUTATIONAL METHODS}

2.1. Classical Molecular Dynamics. The MD simulations were conducted on a cubic simulation cell containing 809 water molecules and a single calcium ion. They were performed with DL_POLY $4.09,{ }^{50}$ which was modified to include a tail required for the SPC/fw-tail force field. ${ }^{27}$ The Verlet leapfrog scheme and the Nosé-Hoover algorithm ${ }^{51,52}$ were used to integrate the equations of motion with a time step of $0.2 \mathrm{fs}$ and to maintain the temperature at $300 \mathrm{~K}$ with $0.5 \mathrm{ps}$ relaxation time. The simulations were carried out in the isothermal-isobaric (constant NPT) ensemble using a NoséHoover thermostat to maintain the average temperature and pressure at $T=300 \mathrm{~K}$ and $P=1 \mathrm{~atm}$. The total simulation time for each of the systems was 144 ns including 200 ps of equilibration. An $8.0 \AA$ cutoff was used for the van der Waals forces, except for the SPC/fw-tail force field in which the cutoff was defined at $9.0 \AA$ with a tail from $6.0 \AA^{27}$

We tested four different force fields to analyze the water dynamics around the calcium cation: first, the shell-model (SM-lj) developed by de Leeuw and Parker and revised by Kerisit and Parker; ${ }^{53,54}$ second, the simple point charge flexible water model (SPC/fw-tail). ${ }^{55}$ Comparison between these first two is the focus of the main paper. However, in order to guarantee the robustness and comparability of these two water force fields, we also included an in-depth assessment of the dynamics and structures produced by SM-lj and SPC/Fw in comparison with two further force fields, that is, SWM4$\mathrm{NDP}^{56,57}$ and TIP3P, ${ }^{58,59}$ which are also commonly used for simulations of calcium-containing systems or in electrolyte solutions. The full assessment of all four force fields can be found in the Supporting Information, which includes a study of the different structural parameters, including the dipole moment, and the three- and four-body order parameters. The dynamics of the system, such as diffusion coefficients and vibrational spectra, can also be found in the Supporting Information, as well as further details and a table with parameters for the different force fields used in this study.

2.2. Ab Initio Molecular Dynamics. AIMD simulations of one $\mathrm{Ca}^{2+}$ in 63 water molecules were conducted with the electronic structure code CP2K/Quickstep version 2.7. ${ }^{60}$ $\mathrm{CP} 2 \mathrm{~K}$ implements the density functional theory (DFT) based on a hybrid Gaussian plane wave. The BLYP, PBE, and revPBE generalized gradient approximations for the exchange and correlation terms were used together with the general dispersion-correction DFT-D3. ${ }^{61-63}$ GoedeckerTeter-Hutter pseudopotentials ${ }^{66}$ were used to describe the core-valence interactions. All atomic species were represented using a double- $\zeta$ valence polarized basis set. The plane wave kinetic energy cutoff was set to $1000 \mathrm{Ry}$. $k$-sampling was restricted to the $\Gamma$ point of the Brillouin zone. Simulations were carried out with a wave function optimization tolerance of $10^{-6}$ hartree that allows for $1.0 \mathrm{fs}$ time steps with reasonable energy conservation. Periodic boundary conditions were applied throughout. Starting from the last configuration of 200 ps of classical MD simulation of 64 water molecules, one $\mathrm{H}_{2} \mathrm{O}$ molecule was replaced by one $\mathrm{Ca}^{2+}$, and we conducted 1 ns of classical MD simulations in the NPT ensemble $(T=300$ $\mathrm{K}$ and $P=1 \mathrm{~atm})$. The last configuration was taken as the starting point for AIMD simulations, which were carried out in the NVT ensemble using a Nosé-Hoover chain thermostat to maintain the average temperature at $T=300 \mathrm{~K}$. The total simulation times for the different functionals were 500 ps for BLYP, PBE, and 40 ps revPBE. For comparison, AIMD simulations of pure bulk water were performed following the same approach.

2.3. Calculations of the Water Exchange Frequencies. To investigate the water dynamics around dissolved calcium ions, the mean residence time (MRT) of water molecules in the first hydration shell of the cation was determined, using both the "direct method" 46 and the Impey method. ${ }^{67}$ An inhouse Python code was developed to calculate the MRT using the direct method from the classical and ab initio MD trajectories (see the Supporting Information). The software tracks all the water molecules that leave or enter the first shell and, subsequently, stay outside or inside the first shell for a time longer than $\tau_{\mathrm{DM}}=0.5 \mathrm{ps}^{46}$ In the direct method (DM), $\tau_{\mathrm{DM}}=0.5 \mathrm{ps}$ is a generally accepted value, based on the average lifetime of a hydrogen bond between the solvent molecules. $^{3,26,46}$ The first hydration shell is taken to be within the distance from the $\mathrm{Ca}$ ion to the position of the first minimum in the $\mathrm{Ca}^{2+}-\mathrm{O}_{\text {water }}$ radial distribution function $(\mathrm{RDF})^{46}$ and is $3.5 \AA$ for all force fields used here (see also Di Tommaso and de Leeuw ${ }^{33}$ for the specific case of calcium). ${ }^{47}$ The inverse of the average exchange frequency is taken to calculate the MRT

$$
\mathrm{MRT}=\frac{t_{\mathrm{sim}} \mathrm{CN}_{\mathrm{av}}}{N_{\mathrm{ex}}^{\tau_{\mathrm{DM}}}}
$$

where $t_{\text {sim }}$ is the total simulation time, $\mathrm{CN}_{\mathrm{av}}$ is the average coordination number for calcium (i.e., the number of water molecules around calcium obtained from integrating the $\mathrm{RDF}$ 
of calcium-oxygen), and $N_{\mathrm{ex}}^{\tau_{\mathrm{DM}}}$ is the number of exchanges counted that lasted longer than 0.5 ps.

The survival function (SF) method (or the Impey method) determines the survival function for water molecules in the first shell. The first shell is defined in the same way as in the direct method, within $3.5 \AA$ from the calcium ion. The SF method measures the residence time (RT) for each water molecule that enters the first shell. RT is recorded only when the leaving time $\tau_{\mathrm{SF}}$ of a water molecule from the first shell meets the minimum time limit. $\tau_{\mathrm{SF}}$ is advised to be set at 2 ps to guarantee that the water molecule truly moved to the bulk, ${ }^{54,67}$ although a $\tau_{\mathrm{SF}}$ of 1 ps has sometimes been used as well. ${ }^{28}$ In this study, we adopt the initial proposal of the method and used $\tau_{\mathrm{SF}}=2 \mathrm{ps}$. After extracting all the residence times for all water molecules around calcium over the entire simulation period, the frequency (occurrences) of each RT is normalized by the total number of RT counted. When plotting the normalized frequencies against time, the survival function is constructed. This function gives the probability that a water molecule stays in the first shell as a function of time. A best fit exponential decay can then be plotted, and the MRT can be calculated by taking the integral of the function:

$$
\operatorname{MRT}=\int_{0}^{\infty} \mathrm{e}^{-r t / M R T} \mathrm{~d} t
$$

\section{RESULTS AND DISCUSSION}

Four different force fields were assessed with respect to structural parameters, diffusion coefficients, and vibrational properties (see the Supporting Information for all details). Here, we focus on the results regarding water dynamics, as reflected by calculated exchange frequencies and mean residence times (MRTs) for water molecules in the first shell around the calcium cation, and the implications for the crystal growth rate.

3.1. Water Dynamics around Calcium Cations. The MRT values obtained using the direct method ${ }^{46}$ and the survival function ${ }^{67}$ are compared with other theoretical and experimental MRT evaluations in Table 1. There are large differences in MRT values obtained with different water force fields while using the same methodology to determine the residence time. For example, $\mathrm{MRT}_{\mathrm{SF}}$ from SM-lj (56 ps) is less than half the one obtained from $\mathrm{MD}$ simulations using the same $\mathrm{Ca}^{2+}-\mathrm{O}_{\text {water }}$ interaction potential but using the SPC/fwbuck water model $(125 \mathrm{ps})$. The longest $\mathrm{MRT}_{\mathrm{SF}}$ is obtained based on the SPC/fw-tail (333 ps). The $\mathrm{MRT}_{\mathrm{SF}}$ value obtained from SM-lj output is the only value to agree with the experimental IQUENS results. The $\mathrm{MRT}_{\mathrm{DM}}$ follows a similar trend where the value obtained from SPC/fw-buck output reproduces the $\mathrm{ab}$ initio results.

It was mentioned in earlier work that the higher number of water molecules in the first shell of calcium in SM-lj causes more exchanges and therefore shorter MRT, suggesting that the higher water density simulated with this force field is responsible for the higher coordination number $(\mathrm{CN})$ of $\mathrm{Ca}^{2+} \cdot{ }^{28}$ We can add that the structure of the water around the cation in SM-lj is also more distorted with a $\mathrm{CN}=8.4$, whereas according to $\mathrm{ab}$ initio $\mathrm{MD}$, the coordination geometry is octahedral $\left(\mathrm{CN}=6^{47}\right)$; the addition of extra molecules probably destabilizes this ideal geometry around $\mathrm{Ca}^{2+}$, contributing to the shorter $\mathrm{MRT}_{\mathrm{SF}}$ for SM-lj.

When comparing the two methodologies used to extract MRT from MD simulations, we note that the values obtained
Table 1. Mean Residence Times Calculated Using either the Direct Method $\left(\mathrm{MRT}_{\mathrm{DM}}\right)$ or the Survival Function $\left(\mathrm{MRT}_{\mathrm{SF}}\right)^{a}$

\begin{tabular}{|c|c|c|c|c|c|c|}
\hline method & $\underset{\mathrm{Ha}^{2+} \mathrm{O}}{\mathrm{N}^{2+}}$ & $\begin{array}{c}\mathrm{MRT}_{\mathrm{DM}} \\
(\mathrm{ps})\end{array}$ & $\mathrm{CN}$ & $\begin{array}{c}\mathrm{MRT}_{\mathrm{SF}} \\
(\mathrm{ps})\end{array}$ & $R^{2}$ & ref \\
\hline SM-lj & 809 & 14.9 & 8.4 & 56 & 0.997 & $\begin{array}{l}\text { this } \\
\text { work }\end{array}$ \\
\hline $\mathrm{SPC} / \mathrm{fw}$-tail & 809 & 116 & 7.0 & 333 & 0.999 & $\begin{array}{l}\text { this } \\
\text { work }\end{array}$ \\
\hline SPC/fw-buck & 809 & 46 & 6.9 & 125 & 0.999 & $\begin{array}{l}\text { this } \\
\text { work }\end{array}$ \\
\hline $\begin{array}{l}\text { BLYP-D3/ } \\
\text { CP2K }\end{array}$ & 63 & 73.8 & 6.8 & & & $\begin{array}{l}\text { this } \\
\text { work }\end{array}$ \\
\hline $\begin{array}{l}\text { revPBE-D3/ } \\
\text { CP2K }\end{array}$ & 63 & 60.2 & 6.1 & & & $\begin{array}{l}\text { this } \\
\text { work }\end{array}$ \\
\hline CPMD & 53 & 23.2 & 6 & & & 47 \\
\hline $\mathrm{QM} / \mathrm{MM}-\mathrm{MD}$ & 199 & 42.5 & 7.8 & & & 3 \\
\hline $\mathrm{SPC} / \mathrm{fw}$-tail & & & & 200 & & 28 \\
\hline $\begin{array}{r}\text { SPC/E/ } \\
\text { TIP4P }\end{array}$ & & & & 158 & & 68 \\
\hline SM-buck & 2027 & 7.0 & & & & 37 \\
\hline $\begin{array}{l}\text { adsorption } \\
\text { exp. }\end{array}$ & & 6310 & & & & {$[*]^{7,8}$} \\
\hline IQUENS exp. & & $<100$ & & $<100$ & & 48 \\
\hline
\end{tabular}

${ }^{a} \mathrm{~N}_{\mathrm{H}_{2} \mathrm{O}} / \mathrm{Ca}^{2+}$ is the number of water molecules per calcium in the simulation cell; the coordination number $(\mathrm{CN})$ was used to normalize the exchange frequency for calculation of $\mathrm{MRT}_{\mathrm{DM}}$, and the correlation coefficient $R^{2}$ is for $\mathrm{MRT}_{\mathrm{SF}}$. [*] Obtained using the exchange frequency of a molecule ${ }^{4,5}$ using equation 1.

using the DM are consistently smaller than those produced by the SF, irrespective of the force field used in the MD simulations (Table 1). Since the definition of the size of the first shell is the same for both DM and SF (3.5 A, see Section 2.3 ), the observed discrepancy can be explained by differences between the two methodologies to calculate (mean) residence times, as well as the different "time constraints" considered $\left(\tau_{\mathrm{DM}}\right.$ versus $\left.\tau_{\mathrm{SF}}\right)$.

In the direct method, an exchange event is counted when a water molecule enters or leaves the first shell around the cation, and this water molecule remains in/outside the first shell for at least $0.5 \mathrm{ps}$, a value for $\tau_{\mathrm{DM}}$ that is commonly used ${ }^{46}$ (see also Section 2.3). The MRT $\mathrm{DM}_{\mathrm{D}}$ is the mean of all residence times (RTs) observed, assuming that the RT distribution is a simple Gaussian distribution. However, the SF method shows that this distribution follows an exponential decay function (Figure 1a), suggesting that the Gaussian distribution is not a valid assumption. As a result, the $\mathrm{MRT}_{\mathrm{DM}}$ preferentially reflects fast exchanging molecules, resulting in shorter values of MRT $_{\mathrm{DM}}$.

In contrast, in the SF method, the $\mathrm{MRT}_{\mathrm{SF}}$ takes into account the actual distribution of normalized RT frequencies. Such a distribution is shown in Figure 1a, based on MD simulations using SPC/fw-tail (SF based on results from SM-lj are shown in Figure S4). The $\mathrm{MRT}_{\mathrm{SF}}$ was calculated by taking the integral of the survival function (i.e., the integral of the fit to these graphs). In our survival functions for all RT probability distributions, a combination of two exponential functions gives the best fit to explain the data (e.g., Figure S6). This fit indicates that two underlying distributions are responsible for the observed $\mathrm{MRT}_{\mathrm{SF}}$, one distribution corresponding to short $\mathrm{MRT}_{\mathrm{SF}}(<20 \mathrm{ps})$ and a second group of long $\mathrm{MRT}_{\mathrm{SF}}(>20 \mathrm{ps})$. A similar biexponential distribution of RTs for the calcium solvation shell was previously reported by De La Pierre et al., ${ }^{28}$ 

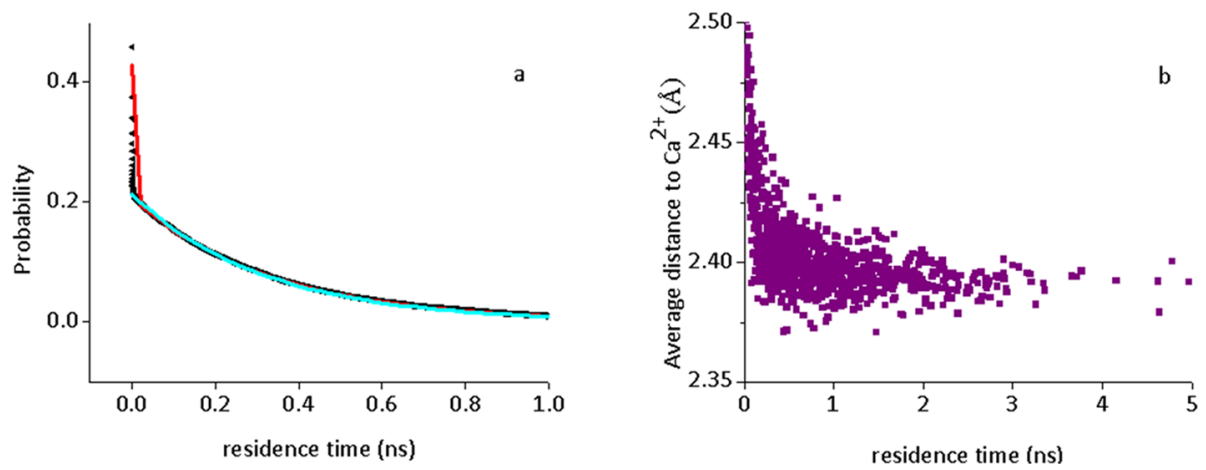

Figure 1. (a) Probability distribution of different residence times (RT, black triangles) for first-shell water molecules obtained using SPC/fw-tail where the survival function is represented by the red and blue lines, which are two exponential fits. (b) Average distance between a coordinated water molecule and the calcium cation as a function of the residence time of that water molecule.

who related the short RTs to water librations and excluded those in their $\mathrm{MRT}_{\mathrm{SF}}$ calculations. Additionally, this bimodal $\mathrm{MRT}_{\mathrm{SF}}$ distribution may be due to differences in the average $\mathrm{Ca}^{2+}-\mathrm{O}_{\text {water }}$ distance. When the average distance is plotted against the RT (Figure 1b), one can see that, while generally all long RTs are found for water molecules at a distance of $\leq 2.40$ $\AA$, short RTs are found for water molecules in the full range of distances, from $2.50 \AA$ to shorter than $2.40 \AA$. This suggests that another property affects $\mathrm{RT}$ besides the $\mathrm{Ca}^{2+}-\mathrm{O}_{\text {water }}$ distance, for example, the $\mathrm{H}$-bonding network. To investigate if this is the case, we determined the percentage of $\mathrm{H}$-bonds between first-shell water molecules and those outside the first shell (referred to as bulk water molecules) for short versus long RT water molecules (Figure 2), following the definition of a hydrogen bond proposed by Chandra. ${ }^{69}$ We used an in-house Python script to count the number of hydrogen bonds in the trajectory file. The molecules having a short RT show a larger number of $\mathrm{H}$-bonds with bulk water molecules than those with long RT, suggesting that the $\mathrm{H}$-bond network may affect residence time as well. Furthermore, SPC/fw-tail shows a larger number of H-bonds with bulk water than SM-lj (Figure 2; Figure S7a). This more pronounced H-bond network around the cation leads to a less disturbed water structure and could therefore explain the longer $\mathrm{MRT}_{\mathrm{SF}}$ obtained from SPC/ fw simulations.

If we group the distance-to-cation data based on their RTs, the average distance of the short RTs is $2.97 \AA$, while the average distance of the long RTs is only $2.48 \AA$ (averaged over

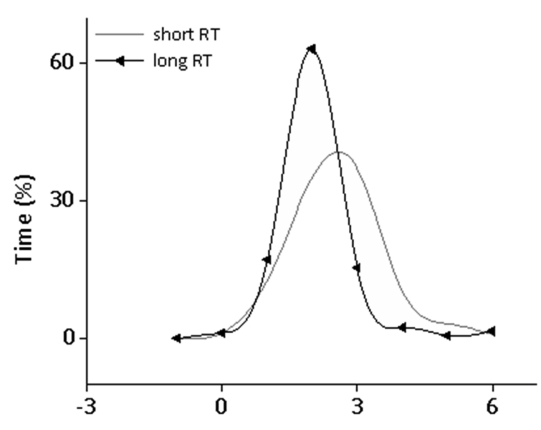

Number of hydrogen bonds with bulk water

Figure 2. Relative time a water molecule has $\mathrm{H}$-bonds with bulk water molecules, during the 20 ps of each residence time (RT); the two gaussians represent the two different populations (short and long RT $\mathrm{H}_{2} \mathrm{O}$ ). the first 20 ps after entering the first shell or, in the case of short RT, water molecules that left the first shell within these 20 ps, as long as they remained).

Besides the $\mathrm{H}$-bond network and $\mathrm{Ca}^{2+}-\mathrm{O}_{\text {water }}$ distance, it is worth considering the structure of the first coordination shell. The average coordination number of SPC/fw water is found to be 7.3 (Table 1). For $96 \%$ of the configurations, the short RT is only observed when the $\mathrm{CN}$ is +1 above this average (Table 2). As mentioned above, $\mathrm{CN}$ is larger for SM-lj (Table 1), and the coordination distances are on average also larger (Figure 3 ), leading to greater distortion of the ideal bipyramid (octahedral) coordination for calcium. Moreover, the width of the first coordination shell is also controlled by the van der Waals interaction when comparing the different interactions using the same water model (SPC/fw-buck and SPC/fw-tail, Figure 3). Using the Buckingham potential and the related parameters leads to a wider first shell, and the first maximum in the RDF is at a slightly larger distance from the cation compared to the tailed Lennard-Jones interaction proposed by Raiteri et al. ${ }^{27}$ Based on the results for both force fields, it may be suggested that longer $\mathrm{Ca}^{2+}-\mathrm{O}_{\text {water }}$ coordination distances reflect first-shell water molecules in excess of the ideal for $\mathrm{Ca}^{2+}$ $(\mathrm{CN}=6)$ that will be more liable to exchange than in the rigid model. Therefore, force fields yielding higher $\mathrm{CN}$ for calcium expectedly give rise to shorter average MRT.

Table 2. Coordination Number of Calcium in Relation to Residence Time of the Water Molecules in the First Shell Using SPC/fw-tail

\begin{tabular}{cccc} 
& \multicolumn{3}{c}{ first shell configuration } \\
\cline { 2 - 4 } $\mathrm{CN}$ & only long RT-water & $1 \geq$ short RT-water & all \\
6 & $1 \%$ & & $1 \%$ \\
7 & $75 \%$ & $1 \%$ & $70 \%$ \\
8 & $25 \%$ & $96 \%$ & $29 \%$ \\
9 & $0 \%$ & $3 \%$ & $0.16 \%$ \\
\hline
\end{tabular}

Both the direct and survival function methods have advantages and disadvantages. The direct method results in shorter mean residence times because the fast exchanges are over-represented. Nevertheless, the relative differences in $\mathrm{MRT}_{\mathrm{DM}}$ we observe between the force fields tested (Table 1) are very similar to those obtained with $\mathrm{MRT}_{\mathrm{SF}}$. Moreover, the DM needs shorter simulation times than SF to obtain statistically meaningful output, and it can therefore also be used to investigate shorter trajectories generated by ab initio 


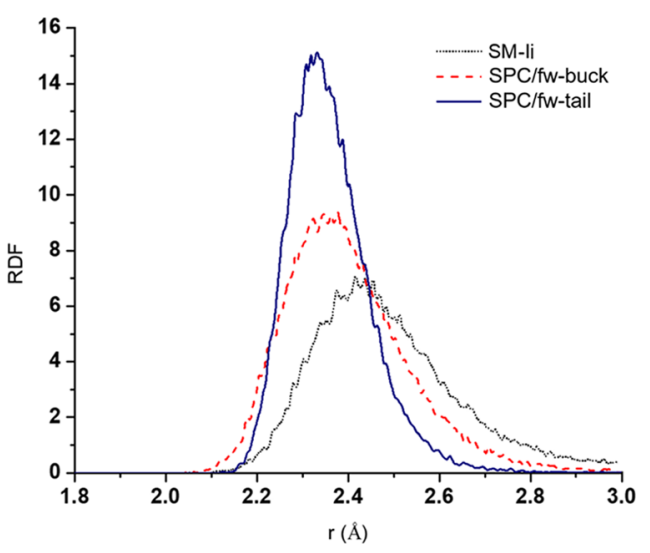

Figure 3. Radial distribution function reflecting $\mathrm{Ca}^{2+}-\mathrm{O}_{\text {water }}$ coordination distances for the different models.

and QM/MM MD methods. However, due to the definition of a water exchange event in the DM method, it is likely to be less accurate. Nonetheless, this method can provide insight into the relative variations in local dynamics, such as a comparative assessment of the reactivity of (i.e., water dynamics around) different ions in aqueous solution or of the change in the reactivity of an ion in aqueous solutions with changing composition. $^{3}$

In contrast, the survival function needs simulation times long enough to capture enough long residence times for the probability distribution function. Depending on the system investigated, this may be hundreds of nanoseconds, even microseconds. However, the SF results facilitate detailed differentiation of the MRT distribution in relation to $\mathrm{CN}$, geometry, and H-bond network, but most importantly, the SF approach closely resembles the data post-processing, in terms of fitting exponential decay functions to determine MRT, used in experiments of Eigen and reviewed by Petrucci. ${ }^{70,78}$ It is also similar to the theory behind the interpretation of IQUENS signals ${ }^{48}$ to obtain MRT from neutron scattering data. As such, the SF results are in principle more comparable to available experimental data for 2 to $3 \mathrm{~m}$ calcium dissolved in water.

When we compare the MRT's extracted from our MD results with those reported from experiments (Table 1), neither methodology (irrespective of the force fields adopted) is able to reproduce the estimation of Eigen. ${ }^{78}$ The MRT values obtained here are closer to IQUENS measurements, ${ }^{48}$ ab initio, ${ }^{47}$ and $\mathrm{QM} / \mathrm{MM}$ MD simulations. ${ }^{46}$ The different computer simulation methods still generate a range of water exchange frequencies, and comparability is hampered (i) because the SF cannot be used on the output generated by computationally demanding AIMD and QM/MM MD methods, which are generally limited to few hundreds of ps, and (ii) partly because of the intrinsic limitations of the classical MD force fields, such as the different ways of expressing the structure of the water with the different interatomic potentials.

The simulations conducted in this study considered a calcium ion in pure liquid water. However, Di Tommaso et al. ${ }^{3}$ have shown that the frequency of water exchange in the first hydration shell of $\mathrm{Ca}^{2+}$ is reduced in the presence of halide ions; this effect is observed not only when the halide anions are directly coordinated to calcium but also when the alkali and halide ions are placed at or outside the second coordination shell of $\mathrm{Ca}^{2+}$. As natural aqueous solutions are far from pure water but rich in ions, in such environments, the water exchange dynamics around $\mathrm{Ca}^{2+}$ is likely to be influenced by the formation of contact and solvent shared ion pairs. An extended model, also considering the effect of solution speciation on the residence times of water around $\mathrm{Ca}^{2+}$, will therefore be part of future studies.

To summarize, residence times are affected by $\mathrm{Ca}-\mathrm{O}_{\text {water }}$ distance and $\mathrm{H}$-bonding network and therefore depend on which force field is used. The direct method can be used on MD as well as AIMD and QM/MM-MD output to obtain relative differences in dynamics. Based on the current comparison, we recommend the SPC/fw-buck force field to determine $\mathrm{MRT}_{\mathrm{DM}}$ since it most closely reproduces the $\mathrm{ab}$ initio results in $\mathrm{MRT}_{\mathrm{DM}}$. However, the $\mathrm{MRT}_{\mathrm{SF}}$ based on this force field is too long compared to experimental IQUENS data where the SM-lj provides the best agreement. Furthermore, if at all feasible, we suggest that the use of the SF method to extract the $\mathrm{MRT}_{\mathrm{SF}}$ from the $\mathrm{MD}$ simulation output obtains the most accurate (absolute) value of the mean residence time.

3.2. Comparison with Water Exchange Kinetics around Carbonate lons. Our atomistic simulations agree with recent experiments, supporting exchange frequency values for calcium that are 2 orders of magnitude larger than previously thought (Eigen). Consequently, the water exchange kinetics around $\mathrm{Ca}^{2+}$ approach has expected values around anions, for example, the carbonate ion $\left(>10^{10} \mathrm{~s}^{-1}\right) .^{37}$ To test this hypothesis, we ran a 13 ns simulation using SPC/FW and carbonate (details in the Supporting Information) to determine the MRT around a carbonate ion. We obtained an $\mathrm{MRT}_{\mathrm{SF}}$ of $8.4 \mathrm{ps}\left(R^{2} 0.999\right)$ and $\mathrm{MRT}_{\mathrm{DM}}$ of 9.2, which are both a factor of 5-40 shorter than for water residence times around calcium obtained with the same force field. As such, the difference in water exchange kinetics around the carbonate ion is not orders of magnitude faster than that around the calcium ion, although it remains noticeably faster. The slightly longer MRT around calcium is expected because the anion interactions are through hydrogen-bonds with a relatively shorter lifetime $(\sim 2.5 \mathrm{ps})^{71}$ than the metal-oxygen interactions as estimated by ab initio MD. ${ }^{47}$ Furthermore, the free energy of hydration for calcium is larger than that for carbonate $\left(-1447 \pm 2\right.$ and $-1301 \pm 2 \mathrm{~kJ} \mathrm{~mol}^{-1}$, respectively), ${ }^{72}$ indicating a stronger interaction between calcium and water than between carbonate and water.

3.3. Implications for Crystal Growth Rate. In order to discuss the impact of a smaller difference in water dynamics around calcium and carbonate ions than has been suggested thus far, we relate to crystal growth via ion-by-ion attachment. While there is strong evidence of ion-pair attachment during growth, for example, by the attachment of $\mathrm{CaCO}_{3}(\mathrm{aq})$ pairs during calcite growth, $5,49,73,74$ such a growth mechanism cannot explain the dependency of the growth rate on the solution stoichiometry at a constant degree of supersaturation observed in bulk ${ }^{75,76}$ and microscopically. ${ }^{17-19}$ According to thermodynamic equilibrium calculations, the concentration of the $\mathrm{CaCO}_{3}(\mathrm{aq})$ ion pair is constant at a constant degree of supersaturation, irrespective of the solution stoichiometry, and the growth step velocity and bulk growth rate dependency on solution stoichiometry are therefore assumed to be caused by an ion-by-ion growth mechanism. ${ }^{16,20-22}$

In the ion-by-ion growth mechanism, the ion attachment frequencies are controlled by several, potentially simultaneous processes: cation and anion diffusion to lattice sites (surface anions and cations, respectively), dehydration of solvated ions, 
and surface lattice sites. Various experiments investigating calcite $^{10-14}$ surface growth strongly suggest that these processes are different for the structurally distinct growth steps at the calcite surface. ${ }^{17-21}$ While obtuse step edges propagate through direct attachment of ions delivered via solution diffusion, the acute steps grow by attachment of ions delivered through surface diffusion. Moreover, Hong and Teng ${ }^{18}$ have proposed, based on their atomic force microscopy observations, that obtuse step growth is limited by calcium dehydration and acute step propagation is limited by surface adsorption, reorientation, and rearrangement of carbonate ions. We would like also to indicate that interpretation of the kinetics of crystal growth, deconvolved from experimental data, using molecular simulations computer models is very challenging because the mechanisms controlling these processes (nucleation and growth) can be significantly more complex than those considered in simplified computer models.

One of the first direct relationships between crystal growth kinetics and ion dehydration was presented by Nielsen. ${ }^{11} \mathrm{He}$ obtained the values for the cation dehydration frequency $\nu_{\mathrm{dh}}$ related to various minerals from Petrucci et al. ${ }^{8}$ and obtained the correlation with ion integration frequency $\nu_{\text {in }}$, as illustrated with blue dots and line in Figure 4. Expressing this correlation

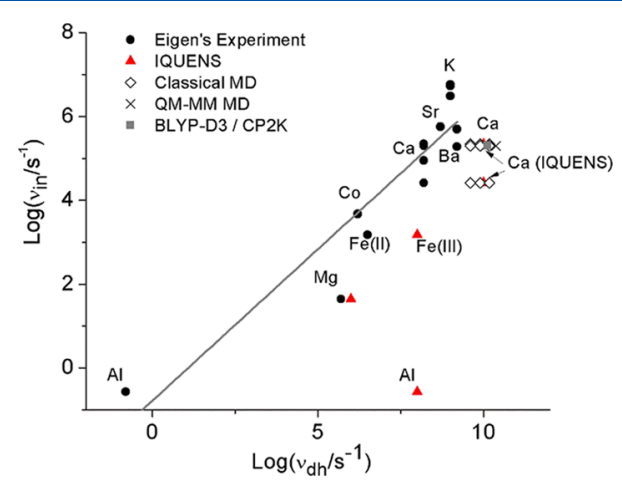

Figure 4. Logarithm of the cation integration frequency versus the logarithm of the cation dehydration frequency. Circles: as determined from parabolic function fitted to the dehydration frequency; ${ }^{11}$ triangles based on IQUENS measurements; ${ }^{48,77}$ cross based on $\mathrm{QM} / \mathrm{MM}-\mathrm{MD}$ simulations and $\mathrm{DM}^{3}$ gray square based on ab initio calculations (BLYP-D3 functional) and DM (this study); diamond from classical MD using SF (this study).

as an Eyring equation, it was found that the integration rate is approximately a thousand times smaller than the dehydration frequency

$$
\nu_{\text {in }}=\nu_{\mathrm{dh}} \mathrm{e}^{-\Delta G_{\mathrm{D}}^{\neq} / k T}=\frac{\nu_{\mathrm{dh}} \nu_{\mathrm{D}}}{\nu_{0}}
$$

where $\nu_{\mathrm{D}}$ is the diffusion frequency, $\Delta G_{\mathrm{D}}^{\neq}$is the Gibbs free energy of diffusion, and the ratio between the diffusion rate and $\nu_{0}=\frac{k T}{h}$, with $k$ and $h$ being the Boltzmann and Planck's constants, respectively, is $\frac{\nu_{\mathrm{D}}}{\nu_{0}}=10^{-3+11}$

This correlation (Figure 4) supported the notion that the cation dehydration is the limiting factor for growth (and dissolution). The cations retain water molecules longer than anions and are therefore rate-limiting. 3,28,37

In general, the MRTs obtained with DM and SF support this notion, although the difference between cation and anion dehydration is not as extreme (orders of magnitude) as considered previously. Both cation and anion dehydration frequencies are in the order of, or slightly smaller than, the reported diffusion rate $\left(\nu_{\mathrm{D}}\right) \sim 10^{10} \mathrm{~s}^{-1}$. $^{11}$ However, adding our water exchange frequencies as well as the other values from Table 1 to Figure 4 reveals that the correlation previously reported by Nielsen ${ }^{11}$ is not very strong, in particular in the case of cations with a dehydration frequency higher than $10^{8}$ $\mathrm{s}^{-1}$ (Figure 4).

Furthermore, if we use the updated values of dehydration frequencies, measured with the IQUENS method, and the values of the ion integration frequency $\nu_{\text {in }}$, then the term $\frac{\nu_{\mathrm{D}}}{\nu_{0}}$ (eq 3 ) is not $10^{-3}$ for calcium anymore, but a factor of $10^{-5}$ is needed to correlate $\nu_{\mathrm{dh}}$ with $\nu_{\mathrm{in}}$. This indicates that another process is affecting $\nu_{\text {in }}$. Furthermore, the dehydration frequency derived from $a b$ initio $\mathrm{MD}$ (Table 1 ) is smaller than the diffusion frequency $\left(\nu_{\mathrm{D}}\right)$, which suggests that the diffusion of the cation into the lattice site from a neighboring position is the rate-limiting step, in agreement with various experimental works. ${ }^{17,18,78}$ Additionally, Petrucci ${ }^{8}$ proposed based among others on data from Eigen and Wilkens ${ }^{7}$ that, for some ions, including $\mathrm{Ca}^{2+}$, dehydration was not the limiting factor. Petrucci concluded that substitution of water by any ligand is the rate-limiting step only if the ligand's exchange rate is less than $10^{7} \mathrm{~s}^{-1}$. The latter is undoubtedly the case for most cations with small radii $(<100 \mathrm{pm})$ such as $\mathrm{Mg}^{2+}, \mathrm{Ni}^{2+}, \mathrm{Co}^{3+}$, $\mathrm{Zn}^{2+}$, and $\mathrm{Fe}^{2+}$. Petrucci ${ }^{8}$ compared the substitution rates of different ligands; for $\mathrm{Mg}^{2+}$, which has a very low water exchange frequency compared to $\mathrm{Ca}^{2+}$, there is indeed no difference in the reaction rate with a range of ligands, indicating that the dehydration of magnesium is the ratecontrolling step. In contrast, he observed different reaction rates between $\mathrm{Ca}^{2+}$ and the same selection of ligands, suggesting that calcium dehydration is not the ratedetermining step. Alternatively, it may also suggest that calcium dehydration can be affected differently by different ligands, as has been shown for halide ions. ${ }^{3}$

To summarize, the water exchange kinetics of $\mathrm{Ca}^{2+}$ and $\mathrm{CO}_{3}{ }^{2-}$ are more comparable than previously thought, which is substantiated by maximum bulk growth rates and step propagation rates in near-stoichiometric solutions. ${ }^{16,75}$ Furthermore, the MRTs reported here, combined with the experimental IQUENS values for the dehydration frequency of calcium, ${ }^{48}$ and the ligand experiments ${ }^{8}$ shed a different light on the most likely rate-limiting step for calcium carbonate crystal growth. Rather than cation dehydration, (surface) diffusion of the cation into the lattice site from a neighboring position is the rate-limiting step, which is in agreement with the work by Hong and Teng, ${ }^{18}$ Bracco et al., ${ }^{19}$ and Stack et al. ${ }^{78}$ Alternatively, our results may support the proposition of De La Pierre et al. ${ }^{49}$ that the attachment of the carbonate ion to the kink site is the rate-determining step of the calcium carbonate crystal growth.

\section{CONCLUSIONS}

The mean residence time of water molecules around dissolved calcium ions, obtained from our classical MD simulations, does not agree with the experimental mean residence time values around calcium estimated by Eigen, ${ }^{7}$ as used in Nielsen's analysis of crystal growth ${ }^{11}$ and adopted by many recent calcite growth rate studies. In contrast, our MRT values are in the same order of magnitude as more accurate $a b$ initio $M D$, whereas $\mathrm{QM} / \mathrm{MM}-\mathrm{MD}$ agrees with the limit obtained from 
IQUENS experiments. This finding indicates that calcium falls in the group of cations with fast water exchanges and its dehydration is therefore not rate-limiting during calcite crystal growth. This study also revealed that, of the two methods we tested to calculate the MRT from MD simulation output, only the survival function method provides accurate absolute values and can distinguish two different groups of water molecules around calcium, revealing that ion-water distance and $\mathrm{H}$ bonding networks affect MRT values. Additionally, our work suggests that the direct method can be used to obtain relative differences in MRT, rather than accurate values, and is most suitable to post-process output from MD simulations of larger systems and ab initio calculations, as it can be implemented on shorter production runs.

\section{ASSOCIATED CONTENT}

\section{S Supporting Information}

The Supporting Information is available free of charge on the ACS Publications website at DOI: 10.1021/acs.jpcc.9b06403.

Tables with the parameters of the force fields used in this work. A study of the different structural parameters including the dipole moment and the three- and fourbody order parameters. The dynamics of the system, such as diffusion coefficients and vibrational spectra for the four water models. A Python code to calculate the MRT using the direct method from the trajectory files. The probability distribution figures for the Ca-SM-lj system (PDF)

\section{AUTHOR INFORMATION}

\section{Corresponding Author}

*E-mail: m.wolthers@uu.nl. Phone: +31302535042.

ORCID

Janou A. Koskamp: 0000-0001-6482-8550

Nora H. De Leeuw: 0000-0002-8271-0545

Notes

The authors declare no competing financial interest.

\section{ACKNOWLEDGMENTS}

The research work of J.A.K. and M.W. is part of the Industrial Partnership Programme i32 Computational Sciences for Energy Research that is carried out under an agreement between Shell and the Netherlands Organisation for Scientific Research (NWO). This work was sponsored by NWO Exact and Natural Sciences for the use of supercomputer facilities. We are grateful to the UK Materials and Molecular Modelling Hub for additional computational resources, which is partially funded by EPSRC (EP/P020194/1). This research utilized Queen Mary's Apocrita HPC facility, supported by QMUL Research-IT. http://doi.org/10.5281/zenodo.438045. Finally, we acknowledge the support of the Supercomputing Wales project, which is part-funded by the European Regional Development Fund (ERDF) via the Welsh Government.

\section{REFERENCES}

(1) Stumm, W.; Morgan, J. J. Aquatic Chemistry : Chemical Equilibria and Rates in Natural Waters; Wiley: 1996.

(2) Nielsen, A. E.; Toft, J. M. Electrolyte Crystal Growth Kinetics. J. Cryst. Growth 1984, 67, 278-288.

(3) Di Tommaso, D.; Ruiz-Agudo, E.; De Leeuw, N. H.; Putnis, A.; Putnis, C. V. Modelling the Effects of Salt Solutions on the Hydration of Calcium Ions. Phys. Chem. Chem. Phys. 2014, 16, 7772-7785.
(4) Kowacz, M.; Putnis, C. V.; Putnis, A. The Effect of Cation:Anion Ratio in Solution on the Mechanism of Barite Growth at Constant Supersaturation: Role of the Desolvation Process on the Growth Kinetics. Geochim. Cosmochim. Acta 2007, 71, 5168-5179.

(5) Andersson, M. P.; Dobberschütz, S.; Sand, K. K.; Tobler, D. J.; de Yoreo, J. J.; Stipp, S. L. S. A Microkinetic Model of Calcite Step Growth. Angew. Chem. Int. Ed. 2016, 55, 11086-11090.

(6) Joswiak, M. N.; Doherty, M. F.; Peters, B. Ion Dissolution Mechanism and Kinetics at Kink Sites on $\mathrm{NaCl}$ Surfaces. Proc. Natl. Acad. Sci. U. S. A. 2018, 115, 656-661.

(7) Eigen, M.; Wilkens, R. G. The Kinetics and Mechanism of Formation of Metal Complexes; American Chemical Society: 1965, $55-80$.

(8) Petrucci, S. Ionic Interactions; From Dilute Solutions to Fused Salts; Elsevier: 1971, 4,

(9) Piana, S.; Jones, F.; Gale, J. D. Assisted Desolvation as a Key Kinetic Step for Crystal Growth. J. Am. Chem. Soc. 2006, 128, 1356813574.

(10) Pokrovsky, O. S.; Schott, J. Surface Chemistry and Dissolution Kinetics of Divalent Metal Carbonates. Environ. Sci. Technol. 2002, 36, 426-432.

(11) Nielsen, A. E. Electrolyte Crystal Growth Mechanisms. J. Cryst. Growth 1984, 67, 289-310.

(12) Lasaga, A. C.; Lüttge, A. A Model for Crystal Dissolution. Eur. J. Mineral. 2003, 15, 603-615.

(13) Lasaga, A. C.; Lüttge, A. Mineralogical Approaches to Fundamental Crystal Dissolution Kinetics. Am. Mineral. 2004, 89, 527-540.

(14) Christoffersen, J.; Christoffersen, M. R. Kinetics of Spiral Growth of Calcite Crystals and Determination of the Absolute Rate Constant. J. Cryst. Growth 1990, 100, 203-211.

(15) Zhang, J.; Nancollas, G. H. Kink Density and Rate of Step Movement during Growth and Dissolution of anABCrystal in a Nonstoichiometric Solution. J. Colloid Interface Sci. 1998, 200, 131145.

(16) Wolthers, M.; Nehrke, G.; Gustafsson, J. P.; Van Cappellen, P. Calcite Growth Kinetics: Modeling the Effect of Solution Stoichiometry. Geochim. Cosmochim. Acta 2012, 77, 121-134.

(17) Bracco, J. N.; Grantham, M. C.; Stack, A. G. Calcite Growth Rates As a Function of Aqueous Calcium-to-Carbonate Ratio, Saturation Index, and Inhibitor Concentration: Insight into the Mechanism of Reaction and Poisoning by Strontium. Cryst. Growth Des. 2012, 12, 3540-3548.

(18) Hong, M.; Teng, H. H. Implications of Solution Chemistry Effects: Direction-Specific Restraints on the Step Kinetics of Calcite Growth. Geochim. Cosmochim. Acta 2014, 141, 228-239.

(19) Sand, K. K.; Tobler, D. J.; Dobberschütz, S.; Larsen, K. K.; Makovicky, E.; Andersson, M. P.; Wolthers, M.; Stipp, S. L. S. Calcite Growth Kinetics: Dependence on Saturation Index, Ca2:CO32Activity Ratio, and Surface Atomic Structure. Cryst. Growth Des. 2016, 16, 3602-3612.

(20) Stack, A. G.; Grantham, M. C. Growth Rate of Calcite Steps As a Function of Aqueous Calcium-to-Carbonate Ratio: Independent Attachment and Detachment of Calcium and Carbonate Ions. Cryst. Growth Des. 2010, 10, 1409-1413.

(21) Larsen, K.; Bechgaard, K.; Stipp, S. L. S. Modelling Spiral Growth at Dislocations and Determination of Critical Step Lengths from Pyramid Geometries on Calcite \{\} Surfaces. Geochim. Cosmochim. Acta 2010, 74, 558-567.

(22) Nielsen, L. C.; DePaolo, D. J.; De Yoreo, J. J. Self-Consistent Ion-by-Ion Growth Model for Kinetic Isotopic Fractionation during Calcite Precipitation. Geochim. Cosmochim. Acta 2012, 86, 166-181.

(23) Larentzos, J. P.; Criscenti, L. J. A Molecular Dynamics Study of Alkaline Earth Metal-Chloride Complexation in Aqueous Solution. J. Phys. Chem. B 2008, 112, 14243-14250.

(24) Rode, B. M.; Schwenk, C. F.; Hofer, T. S.; Randolf, B. R. Coordination and Ligand Exchange Dynamics of Solvated Metal Ions. Coord. Chem. Rev. 2005, 249, 2993-3006. 
(25) Rode, B. M.; Hofer, T. S. How to Access Structure and Dynamics of Solutions: The Capabilities of Computational Methods (Special Topic Article). Pure Appl. Chem. 2006, 78, 525-539.

(26) Wolthers, M.; Di Tommaso, D.; Du, Z.; de Leeuw, N. H. Calcite Surface Structure and Reactivity: Molecular Dynamics Simulations and Macroscopic Surface Modelling of the CalciteWater Interface. Phys. Chem. Chem. Phys. 2012, 14, 15145.

(27) Raiteri, P.; Demichelis, R.; Gale, J. D. Thermodynamically Consistent Force Field for Molecular Dynamics Simulations of Alkaline-Earth Carbonates and Their Aqueous Speciation. J. Phys. Chem. C 2015, 119, 24447-24458.

(28) De La Pierre, M.; Raiteri, P.; Gale, J. D. Structure and Dynamics of Water at Step Edges on the Calcite $\{10 \overline{1} 4\}$ Surface. Cryst. Growth Des. 2016, 16, 5907-5914.

(29) Sena, M. M.; Morrow, C. P.; Kirkpatrick, R. J.; Krishnan, M. Supercritical Carbon Dioxide at Smectite Mineral-Water Interfaces: Molecular Dynamics and Adaptive Biasing Force Investigation of $\mathrm{CO}_{2} / \mathrm{H}_{2} \mathrm{O}$ Mixtures Nanoconfined in Na-Montmorillonite. Chem. Mater. 2015, 27, 6946-6959.

(30) Ruiz Hernandez, S. E.; Streeter, I.; De Leeuw, N. H. The Effect of Water on the Binding of Glycosaminoglycan Saccharides to Hydroxyapatite Surfaces: A Molecular Dynamics Study. Phys. Chem. Chem. Phys. 2015, 17, 22377-22388.

(31) Lee, S. H.; Rasaiah, J. C. Molecular Dynamics Simulation of Ion Mobility. 2. Alkali Metal and Halide Ions Using the SPC/E Model for Water at $25{ }^{\circ} \mathrm{C}$. J. Phys. Chem. 1996, 100, 1420-1425.

(32) Joung, I. S.; Cheatham, T. E., III Molecular Dynamics Simulations of the Dynamic and Energetic Properties of Alkali and Halide Ions Using Water-Model-Specific Ion Parameters. J. Phys. Chem. B 2009, 113, 13279-13290.

(33) Di Tommaso, D.; de Leeuw, N. Modelling the Nucleation of Metal Carbonates: The Importance of the Hydration Shell in thee Monomer Formation. Geochim. Cosmochim. Acta 2010, 74, 236-236.

(34) Pham, T. T.; Lemaire, T.; Capiez-Lernout, E.; Lewerenz, M.; To, Q. D.; Christie, J. K.; Di Tommaso, D.; de Leeuw, N. H.; Naili, S. Properties of Water Confined in Hydroxyapatite Nanopores as Derived from Molecular Dynamics Simulations. Theor. Chem. Acc. 2015, 134, 59.

(35) Hernandez, S. E. R.; De Leeuw, N. H. Effect of Chondroitin 4Sulfate on the Growth and Morphology of Calcium Oxalate Monohydrate: A Molecular Dynamics Study. Cryst. Growth Des. 2015, 15, 4438-4447.

(36) Spagnoli, D.; Cooke, D. J.; Kerisit, S.; Parker, S. C. Molecular Dynamics Simulations of the Interaction between the Surfaces of Polar Solids and Aqueous Solutions. J. Mater. Chem. 2006, 16, 19972006.

(37) Wolthers, M.; di Tommaso, D.; Du, Z.; de Leeuw, N. H. Variations in Calcite Growth Kinetics with Surface Topography: Molecular Dynamics Simulations and Process-Based Growth Kinetics Modelling. CrystEngComm 2013, 15, 5506.

(38) Koishi, A.; Fernandez-Martinez, A.; Ruta, B.; Jimenez-Ruiz, M.; Poloni, R.; di Tommaso, D.; Zontone, F.; Waychunas, G. A.; MontesHernandez, G. Role of Impurities in the Kinetic Persistence of Amorphous Calcium Carbonate: A Nanoscopic Dynamics View. J. Phys. Chem. C 2018, 122, 16983-16991.

(39) de Leeuw, N. H.; Parker, S. C.; Harding, J. H. Molecular Dynamics Simulation of Crystal Dissolution from Calcite Steps. Phys. Rev. B 1999, 60, 13792-13799.

(40) de Leeuw, N. H.; Parker, S. C. Surface-Water Interactions in the Dolomite Problem. Phys. Chem. Chem. Phys. 2001, 3, 3217-3221.

(41) De Leeuw, N. H.; Parker, S. C. Surface Structure and Morphology of Calcium Carbonate Polymorphs Calcite, Aragonite, and Vaterite: An Atomistic Approach. J. Phys. Chem. B 1998, 102, 2914-2922.

(42) Fenter, P.; Kerisit, S.; Raiteri, P.; Gale, J. D. Is the CalciteWater Interface Understood? Direct Comparisons of Molecular Dynamics Simulations with Specular X-Ray Reflectivity Data. J. Phys. Chem. C 2013, 117, 5028-5042.
(43) Marutschke, C.; Walters, D.; Cleveland, J.; Hermes, I.; Bechstein, R.; Kühnle, A. Three-Dimensional Hydration Layer Mapping on the (10.4) Surface of Calcite Using Amplitude Modulation Atomic Force Microscopy. Nanotechnology 2014, 25, 335703.

(44) Söngen, H.; Reischl, B.; Miyata, K.; Bechstein, R.; Raiteri, P.; Rohl, A. L.; Gale, J. D.; Fukuma, T.; Kühnle, A. Resolving Point Defects in the Hydration Structure of Calcite (10.4) with ThreeDimensional Atomic Force Microscopy. Phys. Rev. Lett. 2018, 120, 116101.

(45) Heberling, F.; Trainor, T. P.; Lützenkirchen, J.; Eng, P.; Denecke, M. A.; Bosbach, D. Structure and Reactivity of the CalciteWater Interface. J. Colloid Interface Sci. 2011, 354, 843-857.

(46) Hofer, T. S.; Tran, H. T.; Schwenk, C. F.; Rode, B. M. Characterization of Dynamics and Reactivities of Solvated Ions by $\mathrm{Ab}$ Initio Simulations. J. Comput. Chem. 2004, 25, 211-217.

(47) Di Tommaso, D.; De Leeuw, N. H. First Principles Simulations of the Structural and Dynamical Properties of Hydrated Metal Ions $\mathrm{Me} 2+$ and Solvated Metal Carbonates $(\mathrm{Me}=\mathrm{Ca}, \mathrm{Mg}$, and $\mathrm{Sr}$ ). Cryst. Growth Des. 2010, 10, 4292-4302.

(48) Hewish, N. A.; Enderby, J. E.; Howells, W. S. The Dynamics of Water Molecules in Ionic Solution. J. Phys. C Solid State Phys. 1983, 16, 1777-1791.

(49) de la Pierre, M.; Raiteri, P.; Stack, A. G.; Gale, J. D. Uncovering the Atomistic Mechanism for Calcite Step Growth. Angew. Chem. Int. Ed. 2017, 56, 8464-8467.

(50) Todorov, I. T.; Smith, W.; Trachenko, K.; Dove, M. T. DL_POLY_3: New Dimensions in Molecular Dynamics Simulations via Massive Parallelism. J. Mater. Chem. 2006, 16, 1911.

(51) Hoover, W. G. Canonical Dynamics: Equilibrium Phase-Space Distributions. Phys. Rev. A 1985, 31, 1695-1697.

(52) Nosé, S. A Unified Formulation of the Constant Temperature Molecular Dynamics Methods. J. Chem. Phys. 1984, 81, 511-519.

(53) de Leeuw, N. H.; Parker, S. C. Molecular-Dynamics Simulation of $\mathrm{MgO}$ Surfaces in Liquid Water Using a Shell-Model Potential for Water. Phys. Rev. B 1998, 58, 13901-13908.

(54) Kerisit, S.; Parker, S. C. Free Energy of Adsorption of Water and Metal Ions on the $\{10 \overline{1} 4\}$ Calcite Surface. J. Am. Chem. Soc. 2004, 126, 10152-10161.

(55) Wu, Y.; Tepper, H. L.; Voth, G. A. Flexible Simple PointCharge Water Model with Improved Liquid-State Properties. J. Chem. Phys. 2006, 124, 024503.

(56) Lamoureux, G.; Harder, E.; Vorobyov, I. V.; Roux, B.; MacKerell, A. D., Jr. A Polarizable Model of Water for Molecular Dynamics Simulations of Biomolecules. Chem. Phys. Lett. 2006, 418, 245-249.

(57) Bruneval, F.; Donadio, D.; Parrinello, M. Molecular Dynamics Study of the Solvation of Calcium Carbonate in Water. J. Phys. Chem. B 2007, 111, 12219-12227.

(58) Jorgensen, W. L.; Jenson, C. Temperature Dependence of TIP3P, SPC, and TIP4P Water from NPT Monte Carlo Simulations: Seeking Temperatures of Maximum Density. J. Comput. Chem. 1998, 19, 1179-1186.

(59) Jorgensen, W. L.; Chandrasekhar, J.; Madura, J. D.; Impey, R. W.; Klein, M. L. Comparison of Simple Potential Functions for Simulating Liquid Water. J. Chem. Phys. 1983, 79, 926-935.

(60) VandeVondele, J.; Krack, M.; Mohamed, F.; Parrinello, M.; Chassaing, T.; Hutter, J. Quickstep: Fast and Accurate Density Functional Calculations Using a Mixed Gaussian and Plane Waves Approach. Comput. Phys. Commun. 2005, 167, 103-128.

(61) Lee, C.; Yang, W.; Parr, R. G. Development of the ColleSalvetti Correlation-Energy Formula into a Functional of the Electron Density. Phys. Rev. B 1988, 37, 785-789.

(62) Becke, A. D. Density-Functional Exchange-Energy Approximation with Correct Asymptotic Behavior. Phys. Rev. A 1988, 38, 3098-3100.

(63) Ernzerhof, M.; Scuseria, G. E. Assessment of the PerdewBurke-Ernzerhof Exchange-Correlation Functional. J. Chem. Phys. 1999, 110, 5029-5036. 
(64) Zhang, Y.; Yang, W. Comment on "Generalized Gradient Approximation Made Simple”. Phys. Rev. Lett. 1998, 80, 890-890.

(65) Grimme, S.; Antony, J.; Ehrlich, S.; Krieg, H. A Consistent and Accurate $A b$ Initio Parametrization of Density Functional Dispersion Correction (DFT-D) for the 94 Elements H-Pu. J. Chem. Phys. 2010, $132,154104$.

(66) Goedecker, S.; Teter, M.; Hutter, J. Separable Dual-Space Gaussian Pseudopotentials. Phys. Rev. B 1996, 54, 1703-1710.

(67) Impey, R. W.; Madden, P. A.; McDonald, I. R. Hydration and Mobility of Ions in Solution. J. Phys. Chem. 1983, 87, 5071-5083.

(68) Hofmann, A. E.; Bourg, I. C.; DePaolo, D. J. Ion Desolvation as a Mechanism for Kinetic Isotope Fractionation in Aqueous Systems. Proc. Natl. Acad. Sci. U. S. A. 2012, 109, 18689-18694.

(69) Chandra, A. Effects of Ion Atmosphere on Hydrogen-Bond Dynamics in Aqueous Electrolyte Solutions. Phys. Rev. Lett. 2000, 85, $768-771$.

(70) Eigen, M.; Tamm, K. Schallabsorption in Elektrolytösungen Als Folge Chemischer Relaxation. I. Relaxationstheorie Der Mehrstufigen Dissoziation. Z. Elektrochem. 1962, 66, 107-121.

(71) Kumar, P. P.; Kalinichev, A. G.; Kirkpatrick, R. J. HydrogenBonding Structure and Dynamics of Aqueous Carbonate Species from Car-Parrinello Molecular Dynamics Simulations. J. Phys. Chem. B 2009, 113, 794-802.

(72) Raiteri, P.; Gale, J. D.; Quigley, D.; Rodger, P. M. Derivation of an Accurate Force-Field for Simulating the Growth of Calcium Carbonate from Aqueous Solution: A New Model for the CalciteWater Interface. J. Phys. Chem. C 2010, 114, 5997-6010.

(73) Genovese, D.; Montalti, M.; Otálora, F.; Gómez-Morales, J.; Sancho-Tomás, M.; Falini, G.; García-Ruiz, J. M. Role of $\mathrm{CaCO}^{\circ}$ Neutral Pair in Calcium Carbonate Crystallization. Cryst. Growth Des. 2016, 16, 4173-4177.

(74) Rodriguez-Navarro, C.; Burgos Cara, A.; Elert, K.; Putnis, C. V.; Ruiz-Agudo, E. Direct Nanoscale Imaging Reveals the Growth of Calcite Crystals via Amorphous Nanoparticles. Cryst. Growth Des. 2016, 16, 1850-1860.

(75) Nehrke, G. Calcite Precipitation from Aqueous Solution: Transformation from Vaterite and Role of Solution Stoichiometry. Geol. Ultraiectina; Meded. van Fac. Geowetenschappen, Univ. Utr. 2007, (273).

(76) Tai, C. Y.; Lu, J.-H.; Wu, J.-K. Crystal Growth Rate of Calcite in a Constant-Composition Environment. J. Chin. Inst. Chem. Eng. 2005, 36, 443-450.

(77) Helm, L.; Merbach, A. E. Water Exchange on Metal Ions: Experiments and Simulations. Coord. Chem. Rev. 1999, 187, 151-181.

(78) Stack, A. G.; Raiteri, P.; Gale, J. D. Accurate Rates of the Complex Mechanisms for Growth and Dissolution of Minerals Using a Combination of Rare-Event Theories. J. Am. Chem. Soc. 2011, 134, $11-14$. 